\title{
BLATTVERLUSTE IM SUCHENSINN-KORPUS DER „KOLMARER LIEDERHANDSCHRIFT“
}

\author{
von Michael Bald zuh n, Bochum
}

Der „Kolmarer Liederhandschrift “ (München, Bayerische Staatsbibliothek, Cgm 4997, Sigle t oder, wie nachstehend, $k$ ) ist auf den Blättern $3^{r}-15^{v}$ ein Register vorangestellt, das den Textbestand dieser umfangreichsten wie bedeutendsten Sammlung meisterlicher Lieddichtung des Spätmittelalters systematisch geordnet verzeichnet. ${ }^{1}$ Nach Abschluss der Hauptarbeit auf eine dafür schon früh beiseite gelegte Lage geschrieben ${ }^{2}$, erfasst es in der Reihenfolge der aufgenommenen Autorenkorpora und innerhalb dieser in der Abfolge der Töne jedes einzelne Meisterlied mit seinem Initium, dem jeweils ein Blattnachweis vorangeht und eine Angabe zum Strophenumfang des Liedes folgt. Die neun auf den Blättern $812^{\mathrm{ra}}-813^{\mathrm{vb}}$ und $815^{\mathrm{ra}}-816^{\mathrm{vb}}$ aufgenommenen Lieder $\mathrm{k} 881-885$ und $\mathrm{k} 887-$ 890 des fahrenden, urkundlich 1386, $1389 / 90$ und 1392 in Nürnberg und am Hof Herzog Albrechts II. von Bayern bezeugten Berufsdichters und -sängers Suchensinn ${ }^{3}$ werden im Register $14^{\mathrm{v}}$ in acht Einträgen erfasst. (Das Viererbar $\mathrm{k}$ 888 ist als vermeintliche Dublette zum Dreierbar k 884 übergangen.) An diese acht Einträge schließen $14^{\mathrm{v}}$ aber noch sechs weitere an:

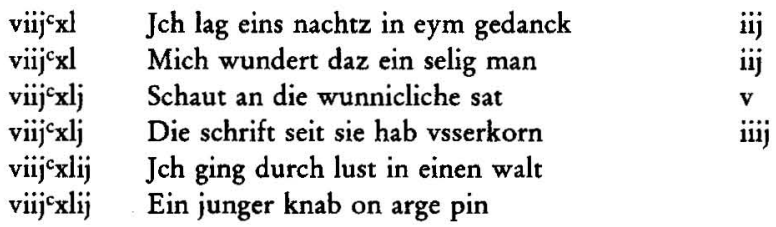

1 Zu k zusammenfassend Burghart Wachinger: ,Kolmarer Liederhandschrift', in: Die deutsche Literatur des Mittelalters. Verfasserlexikon, hg. v. Kurt Ruh [u.a.], Berlin, New York ${ }^{2} 1978 f f .$, Bd. 5, Sp. 27-39. Seither zu ergänzende Forschungsbeiträge bei Lorenz Welker: Kolmarer Liederhandschrift, in: Die Musik in Geschichte und Gegenwart, hg. v. Ludwig Finscher, Kassel [usw.] ${ }^{2} 1994$ ff., Sachteil Bd. 5, Sp. 450-455, hier Sp. 455. Der Textbestand der Handschrift ist einsehbar in: Die Kolmarer Liederhandschrift der Bayerischen Staatsbibliothek München (cgm 4997), in Abb. hg. v. Ulrich Müller, Franz Viktor Spechtler, Horst Brunner, Göppingen 1976 (Litterae 35).

2 Grundlegend zur Entstehungsgeschichte der Handschrift Heinrich Husmann: Aufbau und Entstehung des cgm 4997 (Kolmarer Liederhandschrift), in: DVjs 34, 1960, S. 189243. Wichtige Differenzierungen, die die Vorlagenverhältnisse sowie Datierungs- und Lokalisierungsfragen der Handschrift in ein neues Licht rücken, bei Frieder Schanze: Meisterliche Liedkunst zwischen Heinrich von Mügeln und Hans Sachs, München 1983-84 (Münchener Texte und Untersuchungen zur deutschen Literatur des Mittelalters 82-83), Bd. 1, S. 35-55. Dazu kritisch Christoph Petzsch: Wasserzeichen und Provenienz der ,Kolmarer Liederhandschrift' (Cgm 4997), in: ZfdA 117, 1988, S. 201-210.

3 Zu Autor und Werk zusammenfassend Frieder Schanze: Suchensinn, in: Die deutsche Literatur des Mittelalters (wie Anm. 1), Bd. 9, Sp. 478-481; s. ferner Schanze (wie Anm. 2), Bd. 1, S. 137-145, Bd. 2, S. 26-28; Repertorium der Sangsprüche und Meisterlieder des 12. bis 18. Jahrhunderts, hg. v. Horst Brunner u. Burghart Wachinger unter Mitarbeit v. Eva Klesatschke [u.a.], Tübingen 1986ff. [zit. RSM], Bd. 5, S. 412-423. 
Die drei Blätter 840-842 der hier benutzten römischen Zählung und ihre sechs Lieder sucht man in der Handschrift vergebens, denn deren römische Zählung endet mit 839. Offensichtlich war $k$ also einmal umfangreicher und wurden die Aufzeichnungen noch über das letzte heute erhaltene Blatt $853^{\text {rv }}$ moderner arabischer Zählung und das letzte Lied k 940 in Konrad Harders Hofton hinaus fortgesetzt.

Der Mindestumfang des Verlusts - drei Blätter gibt die noch vom Hauptredaktor angebrachte römische Zählung vor - lässt sich auch aus den vermissten 'Texten errechnen:

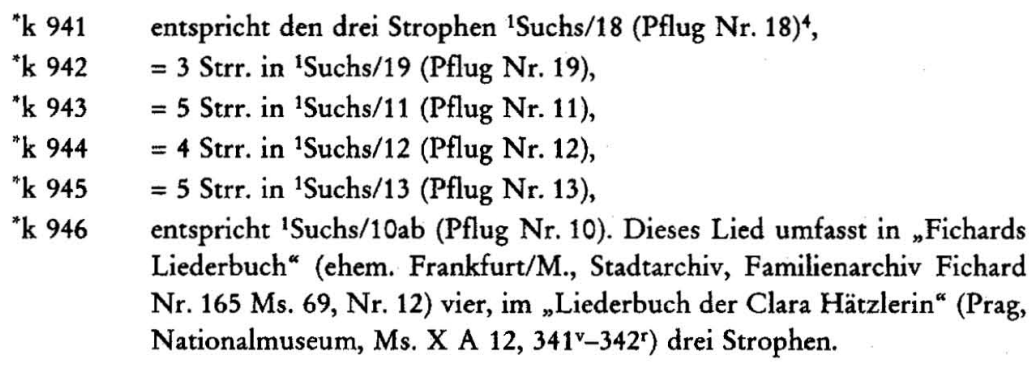

Bei einer in Suchensinns Ton durchschnittlichen Aufzeichnungsdichte von etwa zweieinhalb Strophen pro Spalte, also zehn Strophen pro Blatt, waren für die Niederschrift der fehlenden 23 oder 24 Strophen tatsächlich mindestens drei Blätter erforderlich. Da die Schlusslage 63, die sich über die Blätter 842 bis 853 erstreckt $^{5}$, in sich abgeschlossen ist, kann man sowohl mit einer vollständigen Folgelage rechnen als auch mit drei nur nachgeschalteten Einzelblättern oder zwei Doppelblättern, von denen das letzte frei geblieben sein mochte.

Wann die Blätter entfernt wurden, lässt sich nicht mit Sicherheit klären. Bartschs 1862 erschienenes und bis heute ausführlichstes Verzeichnis liegt bereits der fragmentierte Zustand zugrunde. ${ }^{6}$

Noch vor Bartsch hat der Germanist Christian Friedrich Ludwig Wurm (1801-1861) die Handschrift in München benutzt und unter anderem ein Inhaltsverzeichnis zu erstellen begonnen, das heute im Cgm 5113 aufbewahrt wird. Es reicht aber nur bis Bl. 706 der Handschrift und fällt somit als Kontrollinstrument für den Zustand von k vor Bartschs Ausgabe aus. ${ }^{\text {? }}$

4 Emil Pflug: Suchensinn und seine Dichtungen, Breslau 1908 (Germanistische Abhandlungen 23).

5 Husmann (wie Anm. 2), S. 237f.; Schanze (wie Anm. 2), Bd. 1, S. 39; Karin Schneider: Die deutschen Handschriften der Bayerischen Staatsbibliothek München. Die mittelalterlichen Handschriften aus Cgm 4001-5247, Wiesbaden 1996 (Catalogus codicum manu scriptorum Bibliothecae Monacensis 5,7), S. 424.

6 Meisterlieder der Kolmarer Handschrift, hg. v. Karl Bartsch, Stuttgart 1862 (Bibliothek des Literarischen Vereins in Stuttgart 68), S. 1-89.

7 Vgl. Christoph Petzsch: Die Kolmarer Liederhandschrift. Entstehung und Geschichte, München 1978, S. 189. 
Zudem stammt von Wurm die arabische Bleistiftzählung, die heute allgemein benutzt wird. Sie übergeht das Vorschaltblatt I und läuft dann von 1-853, zählt dabei jedoch 631 doppelt, erfasst also $854(+\mathrm{I})$ Blätter. Eine von Stackmann bemerkte Schlussberechnung auf der letzten verso-Seite der Handschrift ist heute kaum mehr sichtbar, könnte aber, da in Bleistift ausgeführt, ebenfalls von Wurm stammen: ${ }_{n} 856 \mathrm{f}$. / darunter 48 leere f. / - / 808“ ${ }^{8}{ }^{8}$ Merkwürdig bleibt an ihr der Widerspruch zwischen den 853 gezählten und den angegebenen 856 Blättern, obwohl Blatt 853 zum Zeitpunkt der Schlussberechnung doch schon definitiv das letzte der Handschrift gewesen sein muss, da auf ihm ja die Berechnung festgehalten ist. ${ }^{9}$ Das verbietet es, die hier vorliegende Differenz von drei Blättern zwischen 853 und $856 \mathrm{mit}$ den verlorenen Suchensinn-Aufzeichnungen zu verbinden.

Auf der Rückseite des Innendeckels steht in Tinte ${ } 854$ Sk (Rest abgerissen) ${ }^{\star 10}$ : Diese Angabe deckt sich mit dem heutigen Zustand.

Ebenfalls auf dem Rückendeckel steht etwas unterhalb und „von anderer Hand: in $S^{2} 856$ Bll." ${ }^{11}$ Diese Angabe könnte noch den unfragmentierten Zustand bezeichnen - freilich nur dann, wenn sie noch vor der obenstehenden „854 Sk“, also diese Angabe nicht "korrigierend", und unabhängig von der widersprüchlichen Schlussberechnung von $853^{\mathrm{v}}$ angebracht wurde. Das aber ist unwahrscheinlich, denn dann wäre zu erwarten, dass sie auch die 26 Blätter einer Straßburger Meisterliederhandschrift noch mit berücksichtigte, die bis 1857, dem Jahr des Erwerbs der Handschrift für die Münchener Bibliothek, $\mathrm{k}$ beigebunden waren. Diese 26 Blätter wurden erst in München herausgelöst und werden dort heute als Cgm 4999 aufbewahrt. Wahrscheinlicher als die Annahme, die Suchensinn-Blätter wären erst in der Obhut der Staatsbibliothek abhanden gekommen, ist ein Zusammenhang der Angabe ${ }_{n} \mathrm{~S}^{\mathrm{a}} 856$ Bll." mit den in sich widersprüchlichen Angaben vom Schlussblatt $853^{v}$, denen bereits der fragmentierte Zustand zugrunde liegt.

$\mathrm{Zu}$ beachten ist weiterhin ein Briefentwurf Karl Halms vom 25. September 1857, in dem Halm als Leiter der Münchener Staatsbibliothek beim Staatsministerium des Innern für Kirchen- und Schulangelegenheiten für den Ankauf von k wirbt. Dieser Entwurf umfasst auch eine Beilage, die die älteste Beschreibung von $k$ enthält. ${ }^{12}$ Halm gibt diese Beilage als „Mittheilungen [...] über die äußere Form der Handschrift" wieder, die der "Direction der K. Hof- u. St.-B.“ zugekommen seien. Ihre Angaben dürften demnach vom Basler Buchhändler $\mathrm{H}$. Georg verfasst worden sein, von dem die Münchner 1857 ihren Cgm 4997 erwarben. Berichtet werden in dieser Beilage 885 Blätter, von denen aber 26 ursprünglich nicht zugehörige später ange-

8 Die kleineren Dichtungen Heinrichs von Mügeln. Erste Abteilung: Die Spruchsammlung des Göttinger Cod. Philos. 21, hg. v. Karl Stackmann, Berlin 1959 (Deutsche Texte des Mittelalters 50-52), Bd. 1, S. LXV.

9 Auch die Angabe der Leerblätter trifft nicht zu: Schneider (wie Anm. 5), S. 424 zählt nur 46 statt der angegebenen 48.

10 Stackmann (wie Anm. 8), Bd. 1, S. LXV.

11 Ebd.

12 Abgedruckt bei Christoph Petzsch: Die große Kolmarer Liederhandschrift. Ihre Erwerbung im Jahre 1857 durch die Staatsbibliothek in München, in: Mitteilungsblatt der Gesellschaft für bayerische Musikgeschichte München vom 8. Mai 1974, S. 169-200, hier S. $176 f$. 
bunden seien. Mit diesen 26 Blättern ist die schon erwähnte Straßburger Meisterliedersammlung von 1582-91 gemeint. ${ }^{13}$ Deren 26 Blätter werden dann bei den weiteren Berechnungen zum Umfang der Handschrift abgezogen, und ebenso die zwanzig vorangehenden Blätter des Registers, heute $\mathrm{I}+1-19$, so dass der Bericht für die eigentliche Sammlung schließlich auf 839 Blätter kommt - und damit auf vier Blätter mehr als die heute vorliegenden 835 , die es sein müssten, wenn man wie der Bericht zählt und von den 855 heute noch vorliegenden Blättern $(I+854)$ die zwanzig vorangestellten abzieht. Wenn man nicht annehmen möchte, dass diese vier Blätter noch nach ihrem Erwerb für München entfernt wurden, könnten sie $\mathbf{k}$ im Zeitraum zwischen der aus der Wiedergabe Halms bekannten, anpreisenden Beschreibung durch den Basler Buchhändler Georg und der Übersendung der Handschrift von Basel nach München am 14. Dezember $1857^{14}$ entnommen worden sein.

Freilich bleibt gerade vor dem Hintergrund der ohnehin schwankenden älteren Umfangsangaben zu erwägen, ob nicht auch die Abweichungen in Halms Bericht nur auf Irrtümer zurückgehen und die Suchensinn-Blätter bereits bedeutende Zeit früher entnommen wurden. Insbesondere der Zeitpunkt der Ausstattung des Kodex mit einem neuen Einband 1589 und die Beibindung der Straßburger Meisterliederhandschrift von 1582-91 (im Zusammenhang mit der Neubindung?) kommen hier infrage.

Die aus $k$ entfernten Suchensinn-Lieder sind alle schon aus anderen Handschriften bekannt: alle sechs aus „Fichards Liederbuch“, "k 946 zudem aus dem "Liederbuch der Clara Hätzlerin“. Das k-Register vermehrt also lediglich die Text- und Überlieferungszeugen. Diesen Beitrag wird man allerdings nicht geringschätzen: Die Suchsensinn-Überlieferung ist schmal, und die Texte des zweiten Hauptzeugen neben k, des 1944 verbrannten „Fichardschen Liederbuchs", sind nur noch in einem Abdruck von 1815 zugänglich. ${ }^{15}$ Der textkritische Aufschlusswert der Registerinitien bleibt allerdings begrenzt: - das Inzipit von "k 941 weist eine ohnehin offensichtliche Verschreibung in Fichards Handschrift nun als solche definitiv aus ${ }^{16} ;-k$ tritt vor allem mit metrisch glatteren, geregelter alternierenden Texteingängen hervor ${ }^{17}$; - die Strophenordnungen der

13 Beschreibung im RSM, Bd. 1, S. 210 f. Dem Cgm 4999 gehen heute drei römisch I-III gezählte Blätter voran, die als spätere Zutat von der Halm vorliegenden Zählung noch nicht berücksichtigt sind. Der terminus post quem ihrer Vorschaltung ergibt sich aus dem $\mathrm{I}^{\mathrm{r}}$ aufgenommenen Brief des Buchhändlers Georg vom 6. November 1857. Vgl. zum Inhalt der Blätter auch Petzsch (wie Anm. 12), S. 191.

14 Vgl. Petzsch (wie Anm. 12), S. 192.

15 J. C. von Fichard: Altdeutsche Lieder und Gedichte aus der ersten Hälfte des XV. Jahrhunderts, in: Frankfurtisches Archiv für ältere deutsche Litteratur und Geschichte 3, 1815 , S. 196-323.

16 Ich lag eyns tags nachtes in eyme gedanck Fch.] Jch lag eins nachtz in eym gedanck *k 941.

17 Für *k 941 s. Anm. 16. Ferner: Schauwet an die wonnecliche sat Fch. 14] Schaut ... *k 943; Die schrifft die saget sie babe erkorn Fch. 15] D. s. seit sie hab vsserkorn "k 944; Ein junger knabe ane argen pin Fch. 12] ... knab on arge ... "k 946. 
Parallelbezeugung, in der Suchensinn-Überlieferung ohnedies weithin fest ${ }^{18}$, werden nur bestätigt: Die Liedspitzen sind mit den gleichen Strophen wie in den Parallelen besetzt, und wo vom k-Register angegeben, da stimmt auch der Strophenumfang mit den Parallelen überein.

Für die überlieferungsgeschichtliche Einschätzung des Fundes ist der Gesamtbestand des Erhaltenen mitzusehen. ${ }^{19}$

Die fünf von Suchensinn-Nachahmern verfassten Lieder '1Suchs/100-103 und 'Suchs/104, die auch ihre eigenen Überlieferungswege gehen (Basel, UB, Cod. O IV 28 bzw. Weimar, Herzogin Anna Amalia Bibliothek, Fol. 418), sind nachstehend nicht berücksichtigt. ${ }^{20}$

\begin{tabular}{|c|c|c|c|}
\hline k & Fch. & Streuhss. & RSM \\
\hline $\mathrm{k} 881$ & Nr. 13 & Kremsmünster, Stiftsb., Cod. 81, 164"vr(!) & 'Suchs/1 \\
\hline k 882 & & Nürnberg, GNM, Hs. $877,210^{r}-211^{r}$ & ${ }^{1}$ Suchs/2 \\
\hline k 883 & & & ${ }^{1}$ Suchs $/ 3$ \\
\hline k 884 & & & ${ }^{1}$ Suchs/4 \\
\hline k 885 & & & 'Suchs/5 \\
\hline k 887 & Nr. 17 & & ${ }^{1}$ Suchs/6 \\
\hline $\mathrm{k} 888$ & & & ${ }^{1}$ Suchs/7 \\
\hline k 889 & & & ${ }^{1}$ Suchs/8 \\
\hline k 890 & & & ${ }^{1}$ Suchs/9 \\
\hline k 941 & Nr. 22 & & 'Suchs/18 \\
\hline k 942 & Nr. 23/24 & & 'Suchs/19 \\
\hline k 943 & Nr. 14 & & ${ }^{1}$ Suchs/11 \\
\hline k 944 & Nr. 15 & & ${ }^{1}$ Suchs/12 \\
\hline k 945 & Nr. 16 & & ${ }^{1}$ Suchs $/ 13$ \\
\hline \multirow[t]{10}{*}{ k 946} & Nr. 12 & Prag, NM, Ms. X A $12,341^{\mathrm{v}}-342^{\mathrm{r}}$ & ${ }^{1}$ Suchs $/ 10$ \\
\hline & Nr. 18 & & ${ }^{1}$ Suchs/14 \\
\hline & Nr. 19 & & ${ }^{1}$ Suchs $/ 15$ \\
\hline & Nr. 20 & & 'Suchs/16 \\
\hline & Nr. 21 & & 'Suchs/17 \\
\hline & & Sterzing, Stadtarchiv, o. S., $35^{r}$ & ${ }^{1}$ Suchs $/ 20$ \\
\hline & & München, BSB, Clm 8481, $101^{r}-102^{r}$ & ${ }^{1}$ Suchs/21 \\
\hline & & München, BSB, Cgm 1019, 10 $-11^{r}$ & ${ }^{1}$ Suchs $/ 22$ \\
\hline & & Nürnberg, GNM, Hs. $877,214^{\mathrm{v}}-215^{\mathrm{v}}$ & 'Suchs/23 \\
\hline & & Prag, NM, Ms. X A $12,340^{v}-341^{r}$ & ${ }^{1}$ Suchs/24 \\
\hline
\end{tabular}

18 Ausnahmen sind ${ }^{1}$ Suchs/2a+b und 1 Suchs/10a+b: Hier wie dort hat die b-Überlieferung drei Strophen, bewahrt die a-Überlieferung die für Suchensinn typische, aber allgemein ungewöhnlichere Vierstrophigkeit, führt die b-Überlieferung in die Streuhandschriften und die a-Überlieferung in die umfangreicheren Autorsammlungen. In beiden

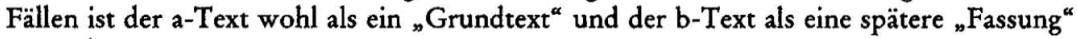
anzusehen.

19 Vgl. zum Folgenden auch die Überlieferungsskizze bei Schanze (wie Anm. 2), Bd. 1, S. 138-141.

20 Vgl. zu diesen Liedern Schanze (wie Anm. 2), Bd. 1, S. 140; Schanze (wie Anm. 3), Sp. 481. 
$\mathrm{k}$ tritt mit nunmehr sechzehn Liedern als die gewichtigste Sammlung von Suchsensinn-Liedern noch deutlicher hervor - zumal Suchensinn in „Fichards Liederbuch", das sich sonst auf Liebeslieder und Reimpaargedichte konzentriert, eine Randstellung einnimmt und dieser Handschrift die einzigen Meisterlieder überhaupt beisteuert. Gemeinsam aber demonstrieren die beiden Überlieferungszeugen (denen vielleicht noch eine weitere Suchensinn-Handschrift, die sich 1444 auf dem Darmstädter Schloss befand, an die Seite zu stellen ist) ${ }^{21}$ die zentrale Bedeutung der Korpussammlung für die Überlieferung spätmittelalterlicher Lieddichtung.

Zwischen diesem Überlieferungstyp einerseits und andererseits der Streuüberlieferung mit je nur einem Lied (Kremsmünster Cod. 81, München Clm 8481, „Sterzinger Miszellaneenhandschrift"; der Cgm 1019 gehört als Meisterliederhandschrift [Sigle y] nur mit Einschränkung hierher) tritt nun aber auch ein dritter Überlieferungstyp etwas deutlicher hervor. Denn $k$ und „Fichards Liederbuch" bauen ihre Korpora unabhängig voneinander auf ( $\mathrm{k}$ vielleicht sogar, wie die Nachstellung der zweiten Liedgruppe ab "k 941 nahelegt, in mehreren Etappen): Die Überschneidungen im Bestand bleiben derart begrenzt, dass keine von $\mathbf{k}$ und "Liederbuch" gemeinsam ausgeschriebene Korpustradition sichtbar wird. Gleichwohl stellen sich immerhin "k 941f. und Fch. 22f. sowie "k 943-945 und Fch. 14-16 zusammen, zwei kleinere Zweier- bzw. Dreiergruppen. Diesen kann man nun auch noch die beiden Liederpaare in der Nürnberger und in der Prager Handschrift beiordnen, die sich je zur Hälfte mit den großen Korpora überschneiden. Jenseits der Streuüberlieferung treten also Lieder in kleineren Gruppen hervor, aus denen die großen Autorenkorpora sich je individuell aufbauen und die im Typ zwischen systematischem Sammelinteresse, das tendenziell auf größere schriftliche Einheiten wie das „Buch“ zielt, und okkasionellpunktuellem Interesse stehen - und damit wenigstens potenziell in größerer Nähe zu Vortragszusammenstellungen kleinerer Gebrauchs-Liederhefte. (Für die umstrittene Echtheit des zweiten Liedes in der Nürnberger Handschrift 877 gibt dieser Überlieferungstyp, der im vorliegenden Fall durchweg in die Nähe des Tonerfinders führt, übrigens ein weiteres Argument für Textautorschaft Suchensinns an die Hand.) ${ }^{22}$

Eine letzte Bemerkung muss der Einordnung der Suchensinn-Sammlung von $\mathrm{k}$ in die Entstehungsgeschichte der Handschrift gelten. Die Lieder wurden offensichtlich in wenigstens zwei Blöcken aufgenommen, zuerst k 881-890 und erst später "k 941-946, und zwar nachdem die Aufzeichnung bereits weiter vorangeschritten und für den unmittelbaren Anschluss an k 890 kein Platz mehr war, ja nirgends mehr Platz außer am Schluss der Sammlung nach k 940, dem letzten Lied des sie heute abschließenden Harder-Korpus. Andererseits kann bei Auf-

21 Vgl. Schanze (wie Anm. 2), Bd. 1, S. 139.

22 Vgl. Schanze (wie Anm. 2), Bd. 1, S. 139f. mit Anm. 14. 
nahme des zweiten Blocks der Registerschreiber noch nicht über $14^{\mathrm{v}}$ mit dem Suchensinn-Teil hinaus gewesen sein. Der Zeitpunkt der Nachtragsaufnahme lässt sich sogar noch genauer bestimmen. Der Platz nach k 940 war zunächst nämlich noch gar nicht für weitere Aufzeichnung frei, vielmehr ursprünglich von den heutigen Lagen 2-6 mit "Prunk-“ und "Sonderformen" (Leichs, Reihen usw.) belegt. Sie wurden nach Ausweis einer arabischen Arbeitszählung erst nach Abschluss der Hauptniederschrift an ihre heutige Stelle zwischen Lage 1 und Lage 7 gestellt. ${ }^{23}$ Erst nach dieser Voranstellung kann der zweite Suchensinn-Block also an die heute vermisste Stelle der Handschrift aufgenommen worden sein.

23 Vgl. Husmann (wie Anm. 2), S. 195-203 und S. 241; Horst Brunner: Die alten Meister. Studien zu Überlieferung und Rezeption der mittelhochdeutschen Sangspruchdichter im Spätmittelalter und in der frühen Neuzeit, München 1975 (Münchener Texte und Untersuchungen zur deutschen Literatur des Mittelalters 54), S. 157-160; Schanze (wie Anm. 2), Bd. 1, S. 42. 